\title{
Structural Characterization of Pt-Bi Thin Films Deposited by Electron Beam Evaporation
}

\author{
X.Z. Li, ${ }^{*}$ P. Kharel ${ }^{*, * *}$, V.R. Shah ${ }^{*}$ and D.J. Sellmyer ${ }^{* * *}$ \\ ${ }^{*}$ Nebraska Center for Materials and Nanoscience, University of Nebraska, Lincoln, NE 68588 \\ ${ }^{* *}$ Department of Physics and Astronomy, University of Nebraska, Lincoln, NE 68588
}

There has been considerable interest in understanding various properties of Pt-Bi based compounds because of their high activity as fuel-cell anode catalysts. Recent reports show that the intermetallic PtBi used as an electrode material exhibits an improved cell efficiency with a dramatic reduction in the Carbon monoxide adsorption in comparison to pure Pt [1,2]. Our investigation on Pt-Bi thin films begins in other approach. As part of a project in the study of transportation and magnetic properties of Bi-Mn-Pt system, thin films of Pt-Bi were deposited by electron beam evaporation and annealed in situ at various temperatures. Our main focus is to understand the structures and electron transport properties of the Pt-Bi thin films [3]. TEM/SAED and XRD studies reveal that the films are composed of the highly textured microstructures of the $\mathrm{PtBi}$ and $\mathrm{PtBi}_{2}$ phases.

$\mathrm{Pt}$ and $\mathrm{Bi}$ were deposited sequentially on glass and silicon substrates at room temperature. The thin films are about $35 \mathrm{~nm}$ of thickness. The data presented here were taken on two samples (A and B) which were prepared at annealing temperature of $300{ }^{\circ} \mathrm{C}$ and $400{ }^{\circ} \mathrm{C}$ respectively. TEM specimens of the sample A were prepared from the fragments of the films on glass substrates which were scratched down and transferred to a TEM Cu grid. Figure 1 shows the TEM image of fragments from sample A. Energy dispersive X-ray spectroscopy (EDS) reveals that the $\mathrm{Pt}$ to $\mathrm{Bi}$ ratio in the sample A is near 50:50. Figure 2 shows the polycrystalline SAED pattern of the sample A (on the left) and the diffraction pattern calculated using PCED2.0 program [4] (on the right). The perfect matching between the experimental and simulated patterns confirms that the sample A consists of the hexagonal PiBi phase.

TEM specimens of the sample B were prepared from mechanical polishing and ion beam thinning. Figure 3 shows the morphology of the sample B deposited on silicon substrate. EDS analysis shows a Pt to Bi average ratio of about 33 to 67. SAED patterns were recorded at different parts of the specimen with series of sample-tilt angles. We have found that the samples contain a mixture of the $\beta-\mathrm{PtBi}_{2}$ and the $\gamma-\mathrm{PtBi}_{2}$ phases. Figure 4 shows $(\mathrm{a}, \mathrm{c})$ the experimental SAED patterns of the sample $\mathrm{B}$ and $(\mathrm{b}, \mathrm{d})$ the corresponding diffraction patterns simulated based on the $\gamma-\mathrm{PtBi}_{2}$ phase using the JECP/ED program [5]. We notice that the sample B also contains a small amount of the hexagonal PtBi phase. As marked by the arrows in figures 4(a) and (c), the weak rings correspond to the PtBi in hexagonal structure. The orientation relationship between the $\gamma-\mathrm{PtBi}_{2}$ and the $\mathrm{PtBi}$ phases is [001] of the $\gamma-\mathrm{PtBi}_{2} / /[001]$ of the $\mathrm{PtBi}$ and (100) of the $\gamma-\mathrm{PtBi}_{2} / /(110)$ of the $\mathrm{PtBi}$.

The XRD results of the samples A and B confirm the conclusions of TEM/SAED studies and also reveal the presence of highly textured microstructures in these films. The XRD patterns of the sample A can be indexed with the hexagonal PtBi phase where its c-axis is in the film plane and those of the sample B can be indexed with the $\gamma-\mathrm{PtBi}_{2}$ phase where its c-axis is out of film plane.

This work was supported by the Nebraska MRSEC (NSF-DMR-0820521), the DOE grant DEFG02-04ER46152 (P.K and D. J. S.), and NCMN. 


\section{References}

[1] E. Casado-Rivera, et al., ChemPhysChem 4 (2003) 193.

[2] M.Oana, et al., Surf. Sci. 574 (2005) 1.

[3] X.Z. Li et al. Phil. Mag., submitted (2011).

[4] X.Z. Li, J. Appl. Cryst. 36 (2003) 956.

[5] X.Z. Li, Ultramicroscopy 110 (2010) 297.

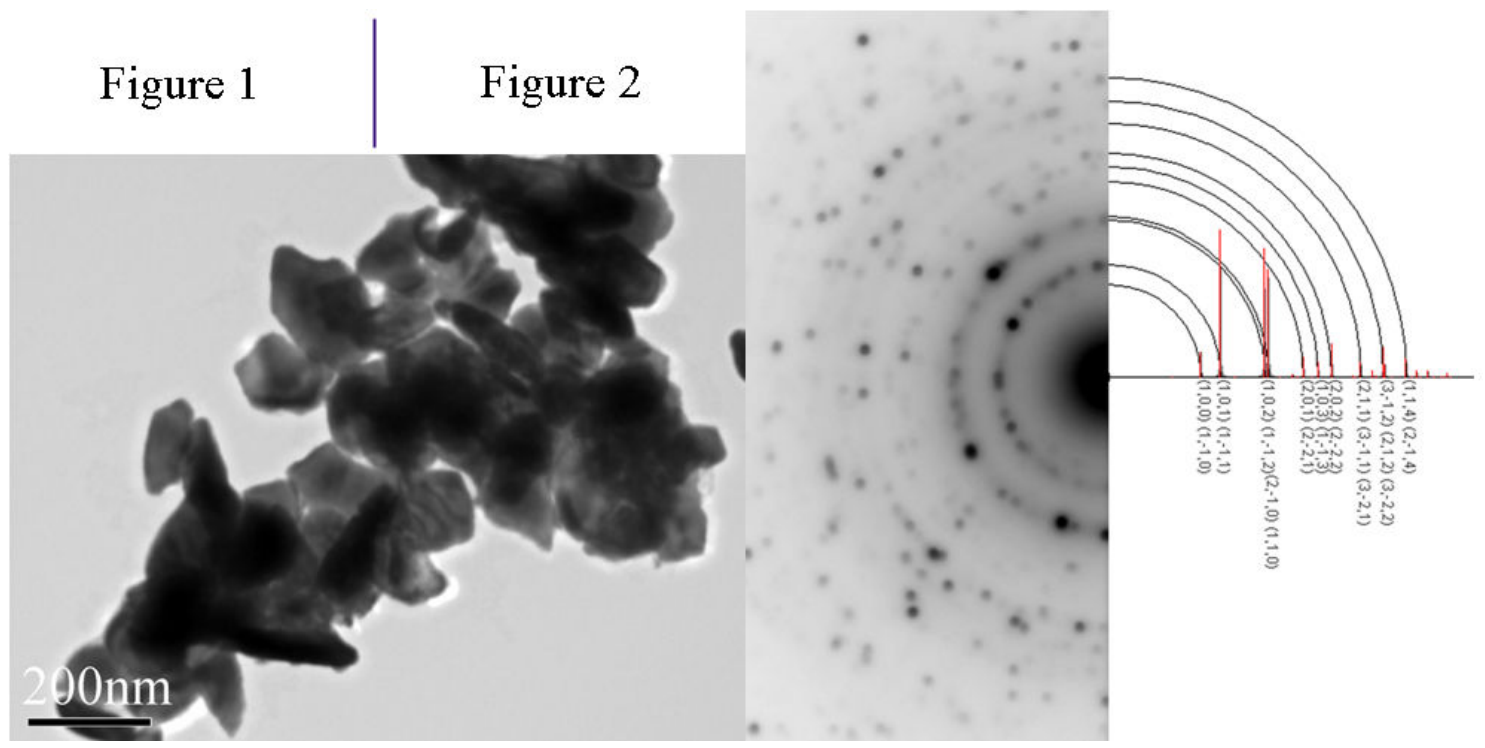

Figure 1. TEM image of the fragments from the thin films deposited on glass substrates and in situ annealed at $300^{\circ} \mathrm{C}$ (sample A). Figure 2. Experimental polycrystalline SAED pattern of the sample A (left side) and the simulated ED pattern based on the structure of the PtBi hexagonal phase.

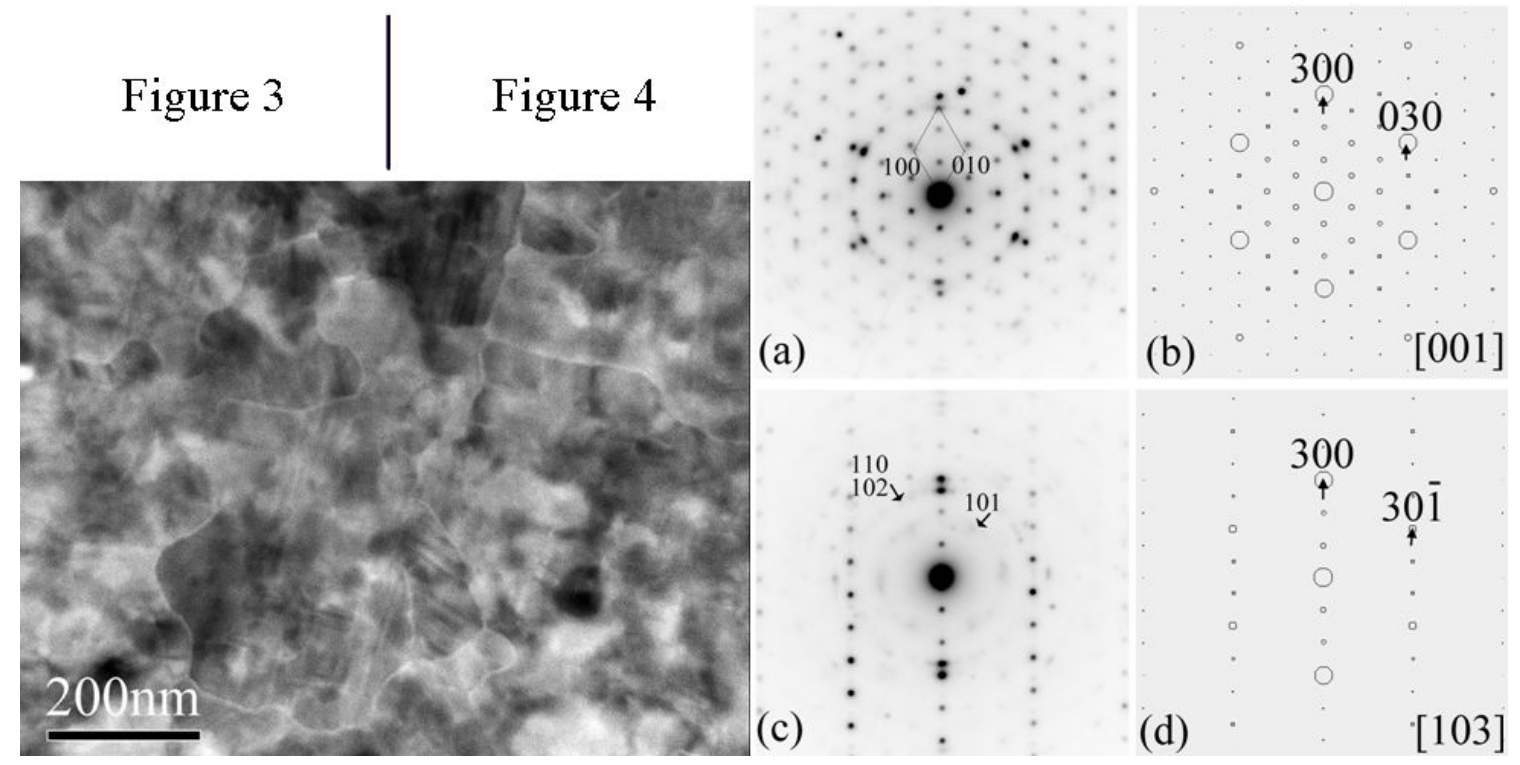

Figure 3. The morphologies of the thin films deposited on Si substrate and in situ annealed at $400^{\circ} \mathrm{C}$ (sample B). Figure 4. Experimental SAED patterns of the sample B, selected from a tilting sample experiment (a and $c$ ) and simulated ED patterns based on the structure of the $\gamma-\mathrm{PtBi}_{2}$ phase (b and $d$ ). The existence of the PtBi phase is marked in the experimental SAED patterns (a and $\mathrm{c}$ ). 